\title{
Characterization of Breast Cancer Radiofrequency Ablation Assisted with Magnetic Nanoparticles: In Silico and in Vitro Study
}

\author{
Silverio Soto Alvarez¹, Lidia Flor E. Huerta1, Alma Verónica Vargas², Jaime López², \\ Jesus Gabriel Silva1, César A. González ${ }^{1,2^{*}}$ \\ ${ }^{1}$ Universidad del Ejército y FAM/EMGS, Laboratorio Multidisciplinario de Investigación, Mexico D.F., Mexico \\ ${ }^{2}$ Instituto Politécnico Nacional, Escuela Superior de Medicina, SEPI, Mexico D.F., Mexico \\ Email: "cgonzalezd@ipn.mx
}

Received 22 October 2015; accepted 18 January 2016; published 22 January 2016

Copyright (C) 2016 by authors and Scientific Research Publishing Inc.

This work is licensed under the Creative Commons Attribution International License (CC BY).

http://creativecommons.org/licenses/by/4.0/

(c) (i) 0 pen Access

\begin{abstract}
The effectiveness of breast cancer ablation by radiofrequency (RF) has been associated to the capacity of concentrating the electromagnetic energy in the tumor region, our group has proposed that this condition could be modulated by appropriate RF exposure cycle times as well as modification of tissue electrical conductivity. The aim of this work was to evaluate analytical and experimental optimal exposure cycle times to induce tissue ablation by RF assisted with magnetic nanoparticles. The study was conducted both analytically by multiphysics simulation of the induced currents in cancer tissue given a magnetron source and experimentally by the observation of hyperthermic effects induced in agar phantoms by a magnetron device by the use of RF assisted with magnetic nanoparticles. The temperature showed significant increases in short periods of time, and was clearly higher under the condition with nanoparticles. Appropriate RF exposure cycle times as well as modification of tissue electrical conductivity by magnetic nanoparticles seems suitable factors to modulate the electromagnetic energy in tumoral regions.
\end{abstract}

\section{Keywords}

Breast Cancer, Radiofrequency Ablation, Multiphysics Simulation, Magnetic Nanoparticles

\section{Introduction}

Breast Cancer (BC) is the most frequent malignancy and the leading cause of death in women worldwide [1]. "Corresponding author.

How to cite this paper: Alvarez, S.S., Huerta, L.F.E., Vargas, A.V., López, J., Silva, J.G. and González, C.A. (2016) Characterization of Breast Cancer Radiofrequency Ablation Assisted with Magnetic Nanoparticles: In Silico and in Vitro Study. Journal of Electromagnetic Analysis and Applications, 8, 1-7. http://dx.doi.org/10.4236/jemaa.2016.81001 
The first therapy option for patients with localized breast cancer is radical mastectomy (surgery) and chemotherapy, radiotherapy and immunotherapy. In the last two decades minimally invasive alternative therapies have been developed, such as cryo-ablation, focused ultrasound high-intensity and radiofrequency ablation (RFA) [2] [3]. Radiofrequency ablation reached importance in recent years for treatment of cancerous tumors. Ablation therapy of tumors by hyperthermia is an application of magnetic nanoparticles, since they have the capacity to produce heat in response to application of external magnetic fields [4]. It is one of the procedures that have gained importance in recent years for the focal destruction of cancerous tumors in general, originally used in the treatment of liver tumors in early 1990s [5] [6]. His clinical potential has expanded significantly in the treatment of breast cancer; however, experimental results of several research groups indicate that the effectiveness of the RFA in the treatment of cancer appears to be associated with the potential ability to focus electromagnetic energy in a specific zone [5]-[7]. Tumors which are smaller than $3 \mathrm{~cm}$ in the greatest diameter are the best candidates for this type of ablation [5]. The aim of this work was to investigate theoretical and experimental optimal characteristics of magnetic fields to produce selective biodegradation effects in tumoral tissue with magnetic nanoparticles concentrations.

\subsection{Biophysical Assumptions}

The induction of currents in conductive materials by oscillating magnetic fields is explained by the induction Faraday's law in terms of general Maxwell differential equations:

$$
\Delta \boldsymbol{B} \times E=-\partial B / \partial t
$$

a magnetic field $\boldsymbol{B}$ variable in time induces an electric potential $E$ in a conductive medium, this potential induces electric currents in the medium (Eddy currents). In addition, an induced current density $J$ in a conductive medium is directly proportional to the electrical potential $E$ and the electrical conductivity of the material as described following equation:

$$
J=\sigma E
$$

Equation above indicates that the absorbed energy by a conductive material is directly proportional to its electrical conductivity [5], and accordingly to the conservation charge law; the energy absorbed will be balanced as a heat dissipation, in this sense we have proposed the use of magnetic nanoparticles to increase the tissue electrical conductivity and concentrate the electromagnetic energy in the tumoral region, this procedure might be considered as focalized RF ablation technique.

\subsection{Radiofrequency Ablation (RFA) and Magnetic Nanoparticles (MNP's)}

Tumor ablation therapy by RF has the potential to be magnified by the use of magnetic nanoparticles (MNP's), since they have the ability to produce heat in response to application of external magnetic fields. The concept of RF tissue hyperthermia assisted by MNP's applies when MNP's are concentrated in the tumor site, and remotely apply heating by magnetic fields in order to reach temperatures up to $55^{\circ} \mathrm{C}$. Ferromagnetic materials generate high heat output and allow the use of magnetic fields at lower frequency and intensity [8]. To the best of our knowledge, there is currently no selective technique that allows ablative process of cancer tissue. Taking advantage of the properties of electrical conductivity and relative permittivity of biological tissues, and based on physical theories of electromagnetism, a biophysical model is proposed to estimate the frequency and optimal exposure time cycles of a magnetic field to promote simulated ablation of cancerous tissue and experimentally tissue hyperthermia by magnetic induction assisted with MNPs.

\subsection{COMSOL Multiphysics}

The mathematical analysis was performed with COMSOL Multiphysics (COMSOL, Inc., Boston, MA, USA, 2005) which employs the finite element method to solve Equation (3) for the magnetic vector potential. Every structural component (subdomain) of the model was meshed in triangular elements. To define boundary conditions the inner electrical parameters of the surrounded sphere correspond to the air and represent electric and magnetic insulation. The process carried out for a modeling using COMSOL Multiphysics is defined by creating a geometry, mesh, physical specifications, the choice of solution and visualization results [9] [10]. 


\subsection{Time-Harmonic, Quasi-Static and Electromagnetic Assumption}

In the time-harmonic case we omit the displacement currents in the Ampere's equation [11]. To simplify initial conditions; we consider a non-moving geometry $(\boldsymbol{v}=0)$ and no external electric potential gradient $(\Delta V=0)$ on the border of the pixel model. In addition, we have assumed that the border of the sphere surrounding the system defined in above section is grounded. Thus, we can obtain:

$$
\begin{gathered}
\left(j \omega \sigma-\omega^{2} \varepsilon\right) \boldsymbol{A}+\nabla\left(\mu^{-1} \nabla \times \boldsymbol{A}\right)=\boldsymbol{J}^{e} \\
\nabla \times\left(\mu_{r}^{-1} \nabla \times \boldsymbol{E}\right)-\mathrm{K}_{0}^{2}\left(\varepsilon_{r}-j \sigma / \omega \varepsilon_{0}\right) \boldsymbol{E}=0, \varepsilon_{r}=n^{2}
\end{gathered}
$$

where $\sigma$ is the medium conductivity (siemens/meter), $\omega$ is the angular frequency (rad/s), $\boldsymbol{J}^{e}$ is a current density generated externally (ampere/meter ${ }^{2}$ ), $\mu$ is the medium permeability (henry/meter), $\varepsilon$ is the medium permittivity (farad/meter) and $\boldsymbol{E}$ is the electric field. In this study equation (3) was solved to obtain the magnetic vector potential ( $\boldsymbol{A})$, a detailed deduction of (3) and its solution is shown in [12].

\section{Materials and Methods}

The study was designed to estimate the induced currents in cylindrical tissue phantoms by a RF magnetron device as well as the involved hyperthermic effect as follows: 1) Theoretical estimation by computer simulation (COMSOL Multiphysics 3.4) and 2) Experimental estimation of hyperthermic effects in agarose phantoms, both conditions by the use of a magnetron device as RF source. Estimations evaluate the presence of tumoral tissue in phantoms under the influence of MNP's. Figure 1 shows a general view of the experimental design.

\subsection{Multiphysics Simulation}

Simulation was developed by the use of the RF module of COMSOL Multiphysics 3.4 (2.45 GHz frequency), and an external current density of $1000 \mathrm{~A}$. Electrical conductivity as well as magnetic flux density were observed in two conditions: Phantom with tumoral tissue and Phantom with tumoral + MNP's. Subdomains description of the simulated model is as follows:

a) Subdomain 1: $29 \times 21 \times 30 \mathrm{~cm}$ cube. Simulates a RF exposure chamber and limits boundary conditions (air).

b) Subdomain 2: Cylinder $4 \mathrm{~cm}$ diameter and $7.4 \mathrm{~cm}$ length. Simulates phantom of healthy tissue.

c) Subdomain 3: Cylinder $1 \mathrm{~cm}$ diameter and $3 \mathrm{~cm}$ length, simulates a RF antenna (magnetron).

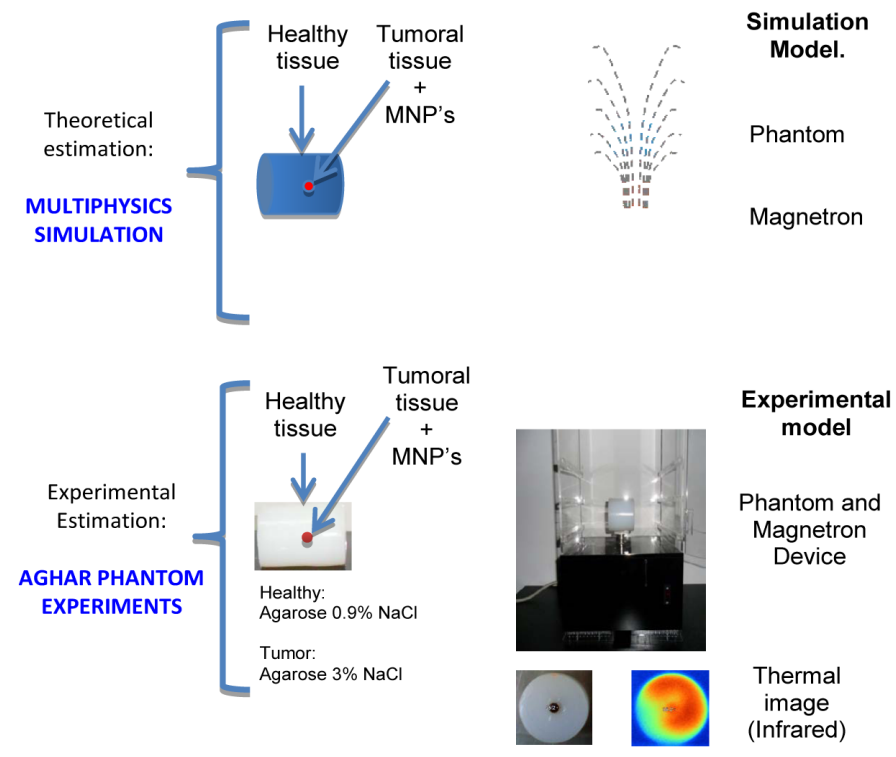

Figure 1. Experimental design for evaluating temperature increases in a cylindrical volume with electrical properties of biological tissues. The oreticaland experimental estimations. 
d) Subdomain 4: Sphere $0.5 \mathrm{~cm}$ radius, simulates phantom of tumoral tissue.

e) Subdomain 5: Spheres of $0.04 \mathrm{~cm}$ radius, simulates concentrations of MNP's.

Respective electrical properties values such as conductivity $(\sigma)$ and relative permittivity $(\varepsilon)$ were assigned to every subdomain and Equations (3) and (4) were solved. Figure 2 showed subdomains of the simulated model.

\subsection{Agar Phantom Design and RF Experiment}

Agar phantoms to emulate cylindrical healthy tissue volumes were designed and built with saline solution (C.S. PISA calve 3608), Agar (Invitrogen Cat No. 30391) and Sucrose (Gibco BRL Cat. No. 5503UA) accordingly to the technique previously described in [13]. In brief, a volume of $200 \mathrm{ml}$ of $\mathrm{NaCl}$ 0.9\%, Agar 5\% and Sucrose 3\% were homogenized at $90^{\circ} \mathrm{C}$ and dropped in a cylindrical model. Additional cylindrical phantoms to emulate tumor volumes of $1 \mathrm{ml}$ were developed with $\mathrm{NaCl}$ 3\% (J.T. Baker, Cat. No. 3624-01) Agar 5\% and Sucrose 3\%, such cylinders were inserted in the posterior side of the cylindrical healthy phantom. Thus; phantoms in three conditions were prepared: 1) Healthy + Tumor + MNPs, 2) Healthy + Tumor and 3) Healthy (Figure 3).

An experimental RF device was designed on the basis of an industrial microwave generator type magnetron (Magnetron-Daewoo Model 2M218 JF, 2.45 1KW GHz) holding in a cubic acrylic chamber specifically designed to accommodate phantoms and minor species (Figure 4). The sequence of RF exposure was determined as 6 cycles of 25 seconds exposure/35 seconds relaxation. The phantoms RF exposure order was randomized and the experiment was developed by triplicate. Thermographic images of the phantoms were acquired with an infrared camera FLUKE® model Ti10, 76,800 pixels, which operates in the infrared spectral band of 7.5 to 14 $\mu \mathrm{M}$ with a thermal sensitivity $\leq 0.045^{\circ} \mathrm{C}$ (Noise Equivalent Temperature Difference-NETD). The images were taken at the same time of day in an environment of temperature and relative humidity of $23^{\circ} \mathrm{C}$ and $40 \%$ respectively, these parameters were monitored for the purpose of operating equipment of thermography under the same conditions only. Thermographic images were taken at a distance of $50 \mathrm{~cm}$ with an isothermal surface background.

\section{Results}

\subsection{Multiphysics Simulation}

The results of multiphysics simulation are shown in Table 1. Increments of magnetic flux density and its induced currents in tumoral tissue as a function of the presence of MNP's are evident. Figure 5 shows the graphic simulation results of magnetic flux density and electrical conductivity in the simulated tissue model.

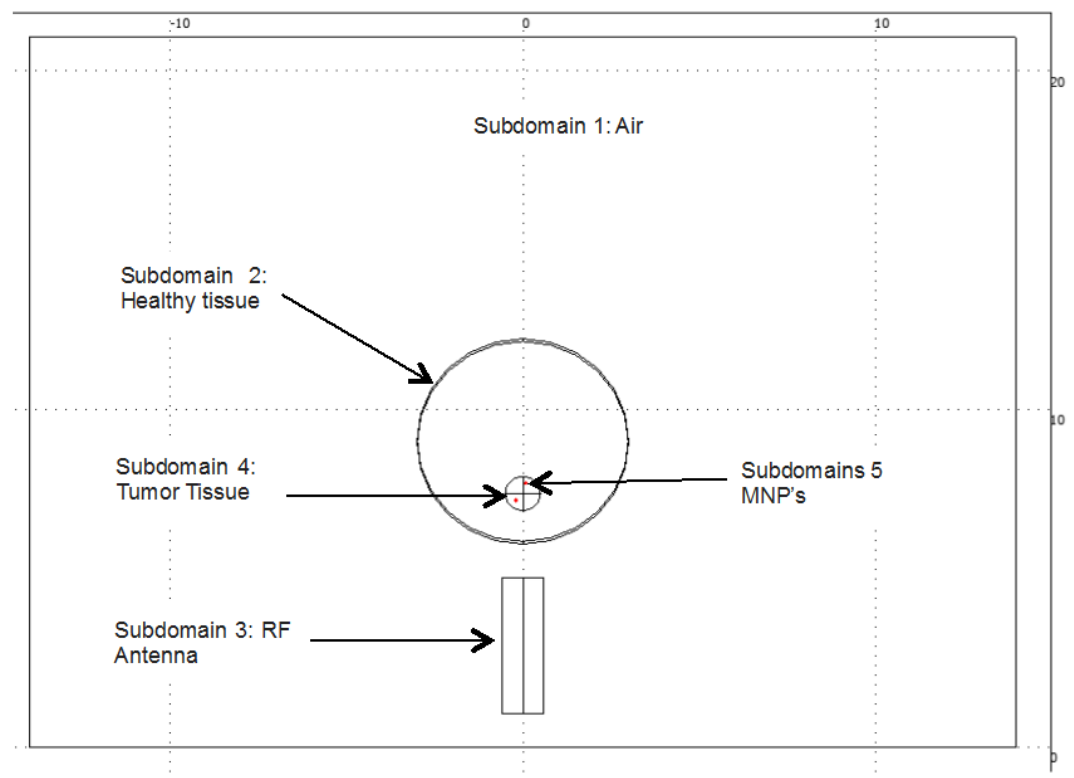

Figure 2. Simulation magnetron in COMSOL Multiphysics 3.4, with their respective subdomains, YZview. 


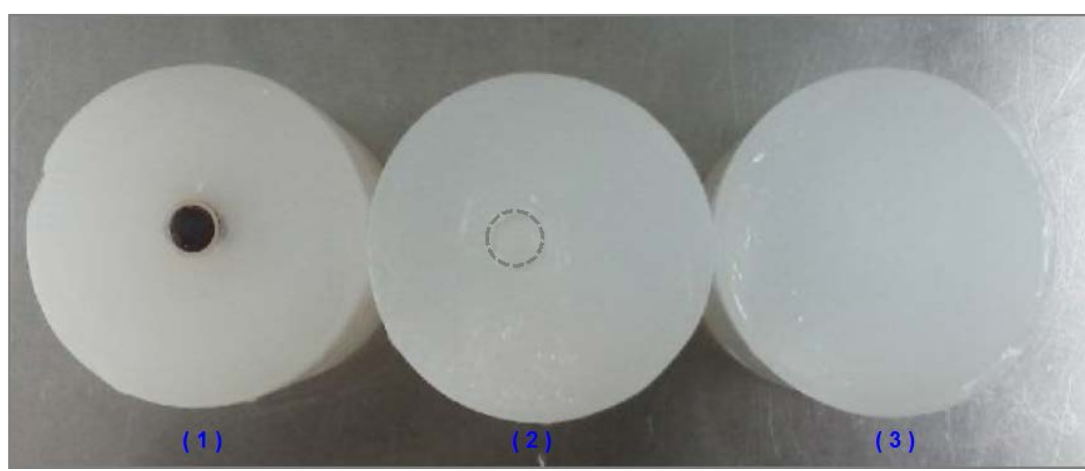

Figure 3. Phantoms prepared in three conditions: 1) Healthy + Tumor + MNPs, 2) Healthy + Tumor and 3) Healthy.

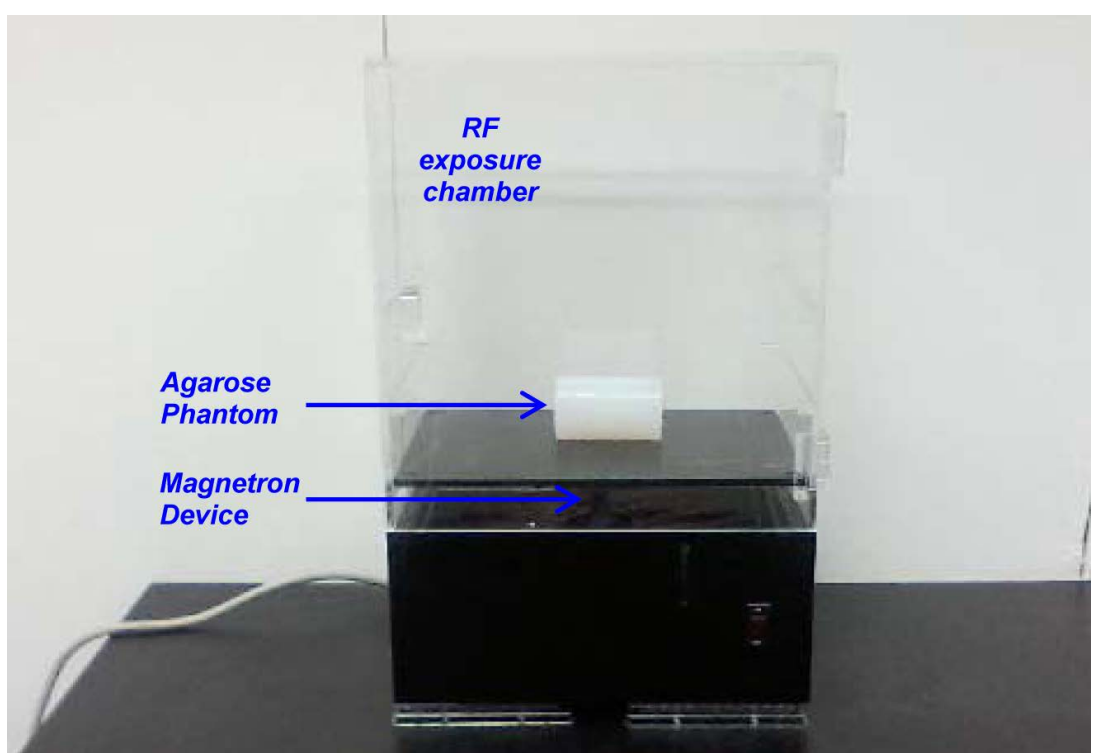

Figure 4. Magnetron device in acrylic holder with a representative agarose phantom.

Table 1. Magnetic fluxdensity estimation.

\begin{tabular}{cccc}
\hline \multicolumn{2}{c}{ Without MNP's } & \multicolumn{2}{c}{ With MNP's } \\
\hline Healthy Tissue & Tumor Tissue & Healthy Tissue & Tumor Tissue \\
$6.610202 \times 10^{-7} \mathrm{~T} \cdot \mathrm{m}^{3}$ & $1.622555 \times 10^{-9} \mathrm{~T} \cdot \mathrm{m}^{3}$ & $6.58401 \times 10^{-7} \mathrm{~T} \cdot \mathrm{m}^{3}$ & $1.744106 \times 10^{-9} \mathrm{~T} \cdot \mathrm{m}^{3}$ \\
\hline
\end{tabular}

\subsection{Experimental Simulation.}

Figure 6 shows the experimental hyperthermia effect of the RF device in phantoms as a function of time (six cycles) and phantom conditions, relevant temperature increments for the Healthy + Tumor + MNP's condition are evident.

\section{Discussion}

The analytical estimations indicate a major concentration of the induced current density lines in the tumor region, the observations suggest that electrical conductivity increments as a function of the presence of MNP's promotes the concentration of the electromagnetic energy in the tumoral region, such condition is in agreement with Faraday's law as previously anticipated in the introduction section. The experimental findings confirm the primary hypothesis of this study, electrical conductivity increment given by the presence of MNP's in the tumoral region 


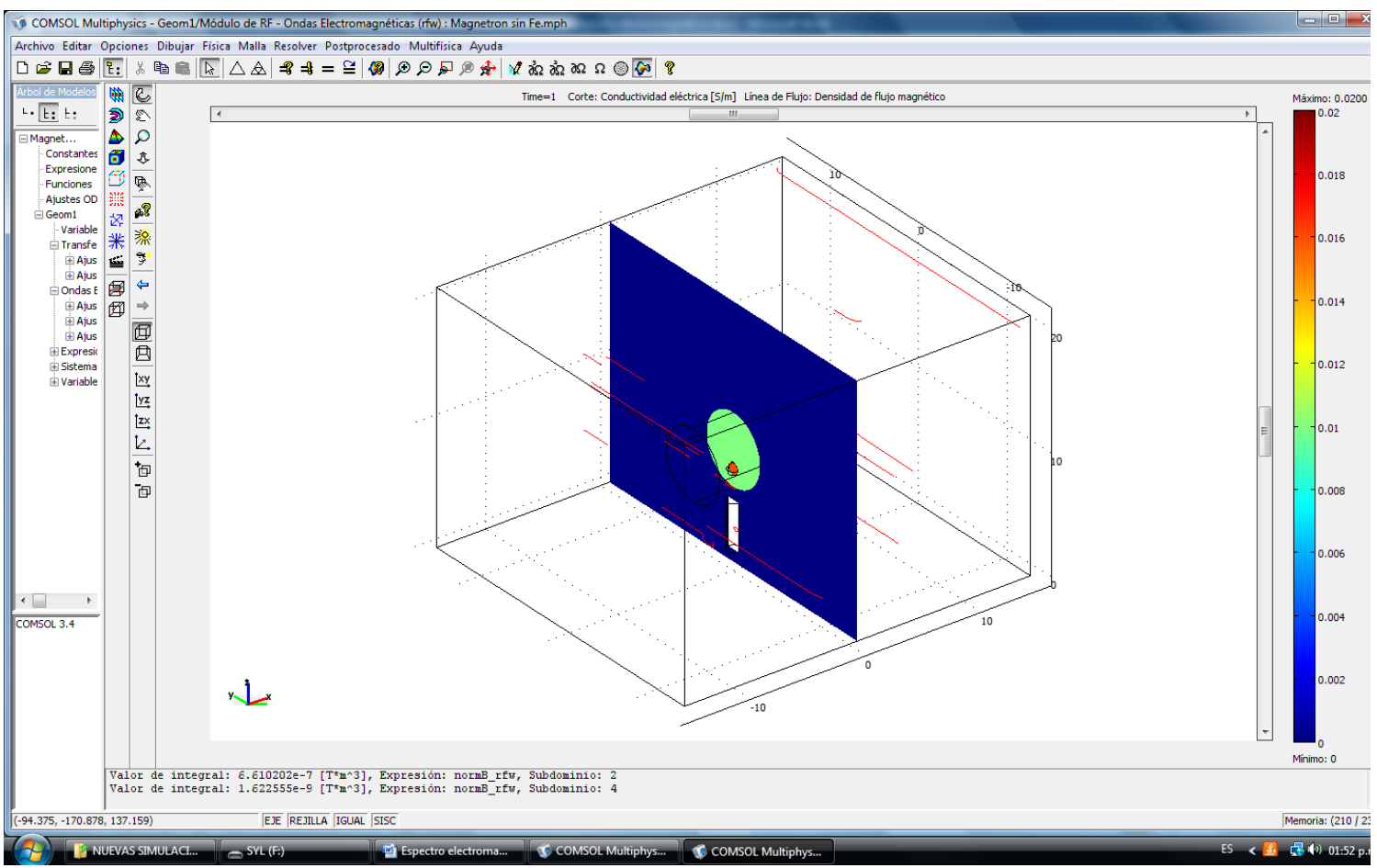

Figure 5. Induction of currentsin biological tissues (simulation), the electrical conductivity in colors, and themagnetic flux density through flow linesis observed.

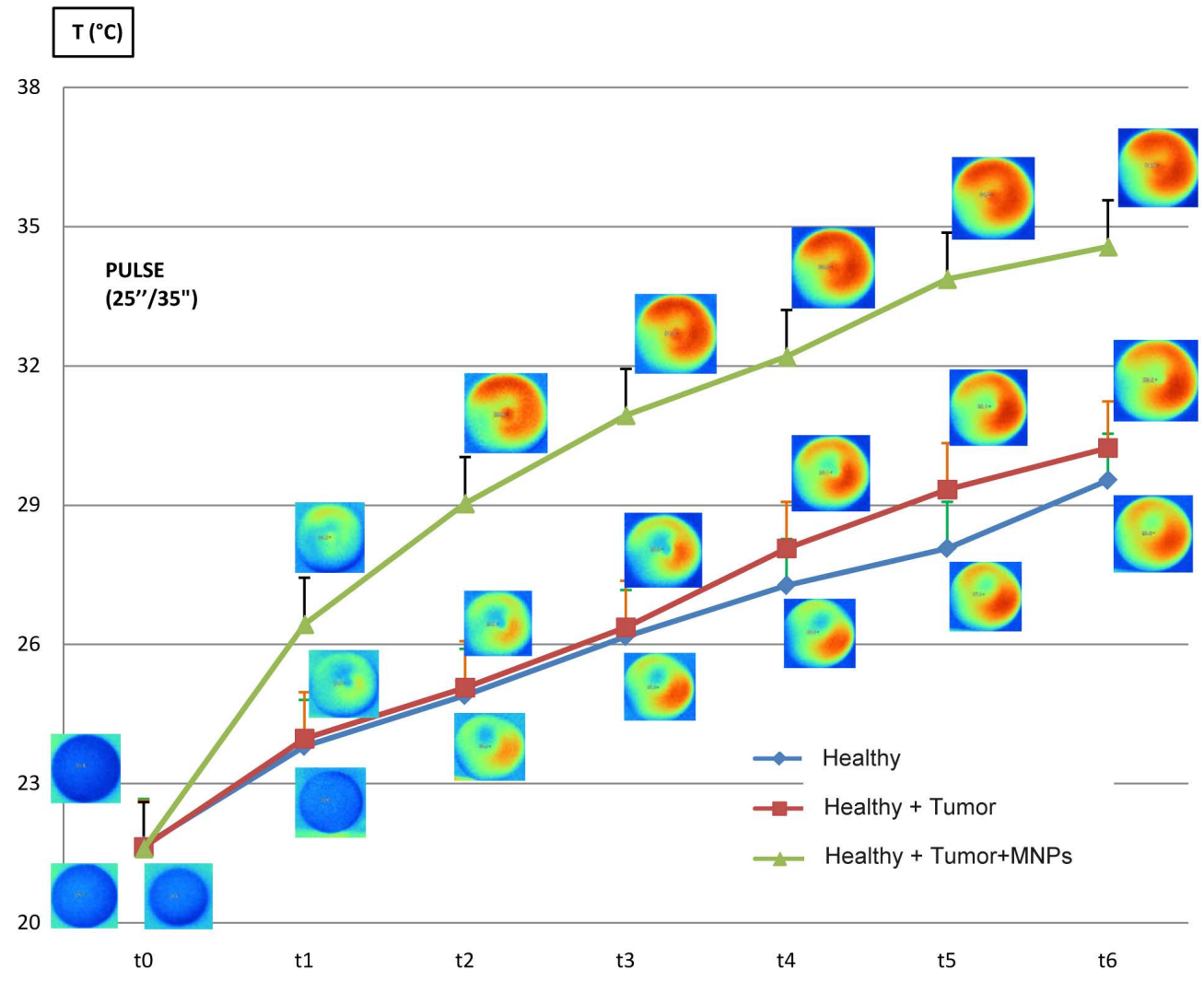

Figure 6. Temperature in crementsin experimental conditions 1) Healthy + Tumor + MNPs, 2) Healthy + Tumor and 3) Healthy. Significative increments in the condition with MNP's as a function of exposure cycles is observed. 
is reflected as focal temperature increments, thus the explored RF exposure cycles (6 pulses of 25”/35” - exposure/relaxation) seem a feasible technique to produce selective biodegradation effects in tumoral tissue with magnetic nanoparticles concentrations.

\section{Conclusion}

The induced current densities and temperature estimated theoretically and experimentally respectively showed significant increments under the explored RF exposure cycles, and were clearly higher under the presence of MNP's. The modulation of RF exposure cycle times as well as modification of tissue electrical conductivity by MNP's seems a feasible technique to concentrate the electromagnetic energy in specific regions and eventually promotes selective ablation of tumoral tissue.

\section{Acknowledgements}

This work was performed in the "Escuela Militar de Graduados de Sanidad-Universidad del Ejército y Fuerza Aérea” (EMGS-UDEFA) Col. Lomas de San Isidro, Miguel Hidalgo, México D.F. C.P. 11620, México, and was supported by “CONACYT CB-2012 under grant No. 180536”.

\section{References}

[1] González, C.A., Uscanga, M.C., Ibarra, C.D., Jiménez, M.E., Lozano, L.M., Silva, J.G., et al. (2012) Differentiation BIRADSI vs II by Magnetic Induction Spectroscopy: Innovative Potential Method for Early Detection of Breast Neoplasms. Research Article. Mexican Journal of Biomedical Engineering, 33, 65-76.

[2] Zagoria, R.J., Hawkins, A.D., Clark, P.E., et al. (2004) Percutaneous CT-Guided Radiofrequency Ablation of Renal Neoplasms: Factors Influencing Success. American Journal of Roentgenology (AJR), 183, 201-207. http://dx.doi.org/10.2214/ajr.183.1.1830201

[3] Hilger, I., Rapp, A., Greulich, K.-O. and Kaiser, W.A. (2005) Assessment of DNA Damage in Target Tumor Cells after Thermoablation in Mice. Radiology, 237, 500-506. http://dx.doi.org/10.1148/radiol.2372041451

[4] Ramos, M. and Castillo, C. (2011) Biomedical Applications of Magnetic Nanoparticles. Concyteg Ideas, 6, 629-646.

[5] González, D.C. (2012) Carcinogenic Ablation Breast Tissue through Radiofrequency Assisted with Bioconjugated Nanoparticles. Research Protocol. IPN School of Medicine Section of Graduate Studies and Research, Mexico D.F.

[6] Florez, C.D., et al. (2012) Radiofrequency Thermal Ablationand the Rapeutic Management of Patients with Lung Cancer. Case Report, Fundación Santa Fe de Bogotá, Bogotá.

[7] Goldberg, S.N., Gazelle, G.S. and Mueller, P.R. (2000) Thermal Ablation Therapy for Focal Malignancy: A Unified Approach to Underlying Principles, Techniques, and Diagnostic Imaging Guidance. American Journal of Roentgenology (AJR), 174, 323-331. http://dx.doi.org/10.2214/ajr.174.2.1740323

[8] Jordan, A., Scholz, R., Maier-Hauff, K., et al. (2006) The Effect of Thermotherapy Using Magnetic Nanoparticles on Rat Malignant Glioma. Journal of Neuro-Oncology, 78, 7-14. http://dx.doi.org/10.1007/s11060-005-9059-z

[9] www.taringa.net/posts/ciencia-educacion/6306379

[10] Mendoza, G.A. (2013) Introduction to the Software COMSOL Multiphysics. Thesis, Faculty of Mechanical and Electrical Engineering, Universidad Veracruzana, Mexico.

[11] Purcell, E.M. (1984) Electricity and Magnetism, Berkeley Physics Course. Berkeley Physics, 2, 263-265.

[12] Gonzalez, C.A., Rojas, R. and Rubinsky, B. (2007) Circular and Magnetron Inductor/Sensor Coils to Detect Volumetric Brain Edema by Inductive Phase Shift Spectroscopy: A Sensitivity Simulation Study. Proceedings of the 13th International Conference on Electrical Bioimpedance and 8th Conference on Electrical Impedance Tomography, Graz, 29th August-2nd September 2007, 315-319. http://dx.doi.org/10.1007/978-3-540-73841-1_83

[13] Solazzo, S.A., Liu, Z., Lobo, M., et al. (2005) Radiofrequency Ablation: Importance of Background Tissue Electrical Conductivity-An Agar Phantom and Computer Modeling Study. Radiology, 236, 495-502. 